\title{
AGENCIAS REGULADORAS FEDERALES DE BRASIL Y ACCESO A LA INFORMACIÓN: ANALISIS DE LOS SERVICIOS DE INFORMACION AL CIUDADANO DE AGENCIAS REGULADORAS BRASILEÑAS
}

\author{
Camila Sanson Pereira Bastos ${ }^{1}$ \\ Diana Santillan Ortega ${ }^{2}$
}

Resumen

El objetivo de ese trabajo es verificar como es garantizado el acceso a la información en las agencias reguladoras brasileñas: "Agência Nacional de Telecomunicações (ANATEL)", "Agência Nacional de Energia Elétrica (ANEEL)" y "Agência Nacional de Petróleo, Gas y Biocombustíveis (ANP)". Esas entidades deben primar por la transparencia y acceso a la información para justificar su legitimidad y cumplir la Ley 12.527/2011, la Ley de acceso a la información (LAI). Es explicado el porqué de la transparencia ser primordial para las agencias reguladoras. Serán analizados de forma cuantitativa y cualitativa los datos proporcionados por el Servicios de Información al Ciudadano (SIC).

Palabras clave: Ley de Acceso a Información; Transparencia; Transparencia pasiva; Agencias Reguladoras; Brasil

\section{FEDERAL REGULATORY AGENCIES AND ACCESS TO INFORMATION: ANALYSIS OF THE CITIZENS INFORMATION SERVICES OF THE BRAZILIAN REGULATORY AGENCIES}

Abstract

The aim of this work is to verify how is provided the access to the information in the Brazilian regulatory agencies: Agência Nacional de Telecomunicações (ANATEL)", "Agência Nacional de Energia Elétrica (ANEEL)" and "Agência Nacional de Petróleo, Gas y Biocombustíveis (ANP)". These agencies have to prioritize the transparency and the access to the information to justify their legitimacy and compliance to Law 12.527/2011, the Brazilian Access to information Law (LAI). Is explained why the transparency should be prioritized by the regulatory agency and the data from the "Service of Information to the Citizens" (SIC) is analysed in a qualitative and in a quantitative way.

Keywords: Access to Information Law; Transparency; Passive Transparency; Regulatory Agencies; Brazil.

\footnotetext{
${ }^{1}$ Mestre en Economía, Regulación y Competencia de los Servicios Públicos por la Universidad de Barcelona. Especialista em Derecho Público por la Universidade do Sul de Santa Catarina - UNISUL. Licenciada en Derecho por la Universidade Federal do Rio de Janeiro - UFRJ.

${ }^{2}$ Mestre en Economía, Regulación y Competencia de los Servicios Públicos por la Universidad de Barcelona. Especialista em Derecho Público Internacional por la Universidad Nacional Autónoma de México - UNAM. Licenciada em Derecho por la Universidad Nacional Autónoma de México - UNAM.
} 


\section{INTRODUCCIÓN}

A partir de la década de los 80 ha empezado a surgir en los países las cuestiones acerca de la reforma del Estado como respuesta a la crisis del Estado intervencionista. En el principio, las reformas buscaban reducir el gasto público y el tamaño del aparato estatal, generando una reestructuración de cómo intervenir y gestionar la estructura administrativa. Mas allá de medidas de control rígido de los gastos, esta primera ola de reformas tuvo como principales resultados concesiones, privatizaciones y formas de cooperación entre gobierno y iniciativa privada y, también, la recolocación del Estado en un papel diferente del anterior. Antes el Estado era visto como ejecutor y después de las reformas pasó a ser encarado como regulador y inductor de las actividades económicas. (REZENDE, 2002)

La Reforma Administrativa fue una de las principales bases de la segunda generación de reformas que tenían en común la necesidad de rever la actitud de la administración pública a través de cambio institucional y ajuste fiscal. Uno de los centros de las discusiones fue la demanda por más participación de la sociedad en supervisión de los servicios públicos.

Así, como resultado de esas reformas, en 1995 fue aprobado el Plano Director (BRASIL, 1995) que recomendaba la revisión de la estructura estatal y el aumento de la gobernanza. Se establecieron cuatro segmentos para que el Estado actúe. El primer segmento es el núcleo estratégico que versa acerca de restablecerse la capacidad del gobierno de formular y evaluar políticas públicas Tanto en el segundo segmento cuanto en el tercer (actividades exclusivas y servicios no exclusivos del Estado), la finalidad es aumentar la eficiencia y eficacia en la prestación de servicios. En el caso de las actividades exclusivas, sector competente para realizar poder impositivo del Estado (reglamentación, fiscalización, seguridad pública, fomento y regulación, etc.) fueron creadas las Agencias Autónomas que comprende las Agencias Ejecutivas y Agencias Reguladoras, esta última será objeto de estudio profundizado en el presente trabajo. En el sector de los servicios no exclusivos del Estado, la propuesta fue ejecutarlos con entidades públicas no estatales (organizaciones sociales y organizaciones no gubernamentales). En el cuarto segmento, de la producción de bienes y servicios para el mercado, constituido de empresas estatales, la propuesta fue la privatización. (PEREIRA, 1996) En ese 
contexto, fueron creadas las primeras agencias reguladoras brasileñas que estaban relacionadas con la privatización y la quiebra del monopolio del Estado en sectores de infraestructura; Agencia Nacional de Energía Eléctrica (ANEEL), Agencia Nacional de Telecomunicaciones (ANATEL) y Agencia Nacional de Petróleo, Gas Natural y Biocombustibles (ANP). (PRADO, 2006) Después, el modelo surgido solo para regular sectores bajo monopolio estatal que pasaban a ser privatizados fue aplicado en otras áreas estratégicas.

En el período de 1999 a 2000, fueron creadas la segunda generación de agencias reguladoras para operar en mercados competitivos y monitorear precios y fiscalizar patrones de oferta y cualidad de los servicios de los entes regulados. Así surgieron la Agencia Nacional de Vigilancia Sanitaria (ANVISA) y la Agencia Nacional de Salud Suplementaria (ANS). (SANTANA, 2002)

Entre 2000 y 2002 ha surgido la tercera generación: la Agencia Nacional de Aguas (ANA), Agencia Nacional del (ANCINE), Agencia Nacional de Transportes Terrestres (ANTT), Agencia Nacional de Transportes Acuáticos (ANTAQ) y en 2006 la Agencia Nacional de la Aviación Civil (ANAC). En esa reforma, se ha perdido el referencial de la primera generación de agencias que era regular de mercados monopolistas que estaban en transición para los mercados competitivos. (SANTANA, 2002)

Las agencias analizadas en el presente trabajo son las de la primera generación ANEEL, ANATEL y ANP porque fueron creadas un contexto de necesidad de transparencia para regular un mercado que antes era monopolio estatal. También porque el formato institucional de esas agencias fue diseñado para garantizar la credibilidad regulatoria (y así, cuidar de la transparencia) principalmente en el área económica, ya que el gobierno, cuando buscaba recursos para pagar la deuda pública, justificaba no poder invertir en esos segmentos. En ese contexto de transparencia, más recientemente ha surgido el debate acerca de la accesibilidad de las informaciones públicas y, en consecuencia, la mejor prestación de cuentas de los gobiernos. Esas cuestiones hicieron con que el tema de la prestación de cuentas democráticas entre en la reforma de como una manera de mejorar el control y la participación en las políticas públicas, pero también como una forma de legitimar el gobierno y las agencias reguladoras. (GRAU, 2000) 
En ese ambiente, junto con la incorporación de la tecnología de información y comunicación por la administración pública, fue publicada la Ley 12.527/2011 (BRASIL,CONGRESSO NACIONAL, 2011), conocida como Ley de Acceso a la Información (LAI). Ese instrumento normativo ha venido para consolidar la transparencia y el derecho de acceso a la información. (BRITO, PERES, et al., 2017)

Así, el presente trabajo busca examinar, en la práctica, como esa Ley está siendo cumplida por las agencias surgidas en la primera generación (ANEEL, ANATEL y ANP) en un escenario de demanda por transparencia. Para eso, se realizará un análisis descriptivo del funcionamiento de los servicios de acceso a la información y un análisis cuantitativo de los pedidos de información recibidos por los órganos independientes en los últimos 5 años (desde mayo de 2012 - cuando ha empezado a funcionar los e-sic hasta abril de 2017). Por lo tanto, será estudiada la transparencia pasiva prevista en la LAI.

La pertinencia de estudiar la transparencia pasiva reside en 3 bases: a) los resultados pueden ayudar a contribuir a un diagnóstico de la transparencia pasiva en las agencias estudiadas; b) la transparencia activa todavía no es considerada por la doctrina como una realidad a pesar de también prevista en la LAI; c) son pocos los estudios acerca de la transparencia pasiva en Brasil. (RAUPP e PINHO, 2016)

Además, para mejor comprensión del tema, serán destacadas la necesidad de trasparencia y acceso a información para las agencias reguladoras y se analizará la Ley de Acceso a Información brasileña.

\section{AGENCIAS REGULADORAS Y TRANSPARENCIA}

En EEUU, Europa y Brasil hubo el reconocimiento de nuevos permisos a la administración pública, en especial, la facultad de intervenir sobre la actividad empresarial de interés general. Ese fenómeno ha generado la creación de organismos con una variedad de poderes con margen de apreciación. El fundamento de esa nueva práctica pública era la demanda de aumentar la credibilidad, enfatizando la neutralidad y previniendo los conflictos de intereses. En general, la doctrina vincula esa nueva regulación con el fin de las restricciones competitivas y de los monopolios que, en la mayoría, eran características de los servicios públicos esenciales. (MALARET, 2014)

En Brasil, como vimos, esas autoridades independientes creadas fueron 
llamadas de agencias reguladoras. La primera generación de las agencias tuvo como justificación el fin de los monopolios en los servicios públicos económicos y, por eso, más una vez se justifica la elección de las tres agencias reguladoras para el análisis en el presente trabajo: ANEEL, ANATEL y ANP.

Es importante afirmar que las decisiones reguladoras importantes deben basarse en informaciones cualificadas y debe ser transparente a la población. En especial, los reguladores deben justificar sus decisiones con la mejor información técnica y científica. (BEALES, J., et al., 2012) De ese concepto, se pueden extraer dos características de las agencias reguladoras, la especialización y la transparencia.

La especificidad es originada de la necesidad de un saber profesional específico para que la autoridad justifique su decisión de la manera más fundamentada. La transparencia es necesaria para legitimar la existencia de las autoridades independientes una vez que son autoridades administrativas autónomas fuera del poder de dirección gubernamental En ese entorno, la transparencia tiene mucha relevancia y debe ser interpretada como publicidad y claridad en los procesos de decisiones. Así, esta transparencia es fundamental para completar la legitimidad de la democracia basada en el principio electivo-representativo. La credibilidad, la fiabilidad y la confianza de las decisiones traen seguridad jurídica y el funcionamiento correcto de los mercados. Con eso, gobernar "a luz del sol” es una exigencia primaria. (MALARET, 2014)

La transparencia puede ser activa o pasiva, siendo concretada en el ordenamiento jurídico como principio general. (DÍAZ, 2014) La transparencia activa es cuando la revelación de la información ocurre como una iniciativa de la administración. Esta es verdadera cuando se basa en datos compatibles y reutilizables, y toda la información necesaria para conocer y controlar el funcionamiento estatal. (ETZIONI, 2010) La transparencia pasiva ocurre cuando el ciudadano tiene la iniciativa de solicitar la información al órgano.

En la Ley de acceso a la información de Brasil, son previstas las obligaciones de sumisiones a las dos clases de transparencia. En este trabajo, será analizada la vertiente de la transparencia pasiva, que, por la Ley brasileña, como se verá en el próximo apartado, es controlada por el Sistema de Información al Ciudadano (e-SIC).

Asegurar la transparencia significa identificar lo que es público y lo que es privado (es una excepción prevista en el ordenamiento para tutela de algún bien o valor 
constitucional). Por eso, el derecho de acceso a la información es pilar del modelo actual de administraciones públicas, que renueva la legitimidad. (MALARET, 2014)

El derecho de acceso a la información pública debe ser considerado un derecho fundamental por ser imprescindible para la sociedad democrática. En Brasil, incluso, la doctrina (NOVELINO, 2011) argumenta que es esa especie de derecho es derecho fundamental por estar de acuerdo con el artículo 19 de la Declaración Universal de los Derechos Humanos. (ONU, 1948) El individuo tiene el derecho de conocer cómo actúan los poderes públicos, de saber si su actuación es de acuerdo con el mandato democrático que le fue conferido. (MAÑAS, 2014)

El derecho de acceso no responde sólo a la defensa de intereses de un individuo, también es cuestión de conocimiento de la documentación e información que dispone la administración. Este derecho de acceso a la información debe ser garantizado de manera adecuada y coherente. (MALARET, 2014)

El objetivo de este trabajo es verificar como está siendo garantizado el acceso a la información en las agencias reguladoras estudiadas (que deben primar por la transparencia y acceso a la información para justificaren su legitimidad). En el próximo apartado, como contextualización, será analizada la Ley de acceso a la información de Brasil.

\section{LEY DE ACCESO A LA INFORMACIÓN}

La evolución mundial de las leyes de acceso empieza en el siglo XIX, cuando Suecia adopta su marco legal, en 1766. En 1888, Colombia se ha convertido en el primer país de Latinoamérica en publicar un decreto que garantiza el acceso a la información. En 1966, los Estados Unidos publicaron la "Freedom of Information Act (FUE A)". (MICHENER, 2011) En la Europa oriental, las leyes de acceso a la información fueron publicadas cuando estaban volviendo a ser una democracia. En España, la Ley de Transparencia, Acceso a la Información Pública y Buen Gobierno (Ley 19/2013 - LTAIP) es de 2013.

La historia del acceso a la información en Brasil es dividida en cuatro etapas legislativas que llevaron a la consolidación de la transparencia pública en Brasil (19341980). Los actos normativos eran relacionados con el escenario que ha posibilitado el 
estado burgués que, en la práctica, no tenían mecanismos de acceso a la información. La segunda dimensión ha ocurrido cuando la transparencia pasó a ser instrumento oficial del Estado (1981-1989). En ese período se destaca la Constitución Federal de 1988. (BERNARDES, SANTOS e ROVER, 2015)

En la Constitución, artículo 5, se destaca la mención genérica al acceso a la información (inciso XIV), el deber de transparencia del Estado (inciso XXXIII), referencia al habeas data - que busca asegurar la obtención de informaciones acerca de quien le propone y que existan en las bases de datos del Poder Público - (inciso LXXII). (GUIMARÃES, MOLINA e SANTOS, 2017)

Entre 1989 y 1999 ha ocurrido la tercera dimensión que fue marcada por la gran cantidad de legislación relacionada con la transparencia. Finalmente, la cuarta dimensión ha ocurrido entre 2000 y 2012 y es relacionada con la incorporación de tecnologías y comunicación por la administración. Esa generación puede ser dividida en dos periodos; de 2000 a 2004 cuando hay la necesidad de informar y, después de 2004 cuando el ciudadano es visto como coproductor de las políticas públicas. Justamente en ese segundo momento, en 2011, fue promulgada la Ley de acceso a la Información (LAI), apoyada por la participación de los ciudadanos y por la transparencia. Posteriormente, en 2012, con el Decreto 7724/2012 (BRASIL,CONGRESSO NACIONAL, 2012), fue reglamentada la Ley para el ámbito del Poder Ejecutivo Federal y los e-sic empezaron a funcionar. (BERNARDES, SANTOS e ROVER, 2015)

La Ley brasileña fue considerada una de las 15 leyes de acceso más rigurosas del mundo por la entidad Right to Information Rating do Center for Law and Democracy, la Ley de acceso busca conceder a los ciudadanos la información pública. Ese derecho de acceso puede ser segregado en de forma: (a) activa (prevista en el artículo 8 de la Ley) o (b) pasiva. (BRAGA, 2014). Como resaltado, en el presente estudio, será analizada la forma pasiva de acceso a la información, a través de los e-SICs.

Son sujetos a la Ley de Acceso, por tanto, obligados a fornecer la información en regla, los órganos públicos integrantes de la administración directa de los Poderes Ejecutivo, Legislativo, incluyendo las Cortes de Cuentas, y Judicial y del Ministerio Público; los municipios, las fundaciones públicas, las empresas públicas, las sociedades de economía mixta y demás entidades controladas directa o indirectamente por la Unión, Estados, Distrito Federal y Municipios; las entidades privadas sin fines de lucro que 
reciban, para realización de acciones de interés público, recursos públicos directamente del presupuesto o mediante subvenciones sociales, contrato de gestión, término de asociación, convenios, acuerdos, ajustes u otros instrumentos congéneres.

Los principales aspectos de la LAI son: a)el acceso a la información es la regla, el sigilo es una excepción que debe estar prevista en Ley o en el Decreto 7724/2012; b) Quien requiere la información no necesita declarar el porqué de pedir ni para que lo pide, no hay que motivar el pedido; c) Las hipótesis de sigilo son limitadas por ley (hay limitación del sigilo); d) La concesión de la información es gratuita, salvo el valor de la reproducción (gratuidad de la información); e) Las informaciones de interés colectivo y general deben ser divulgadas (transparencia activa); f) Creación de procedimientos y plazos que facilitan el acceso a la información (transparencia pasiva).

La Ley abarca toda la información producida o guardada por el Poder Público que son públicas y, por tanto, accesibles a todos los ciudadanos, excepto la información de carácter personal (relacionada con una determinada persona. Su tratamiento debe hacerse de forma transparente y respetando la intimidad, la vida privada, el honor y la imagen, así como las libertades y garantías individuales.) e hipótesis de sigilo establecidas legalmente.

Las informaciones clasificadas como sigilosas son aquellas que la divulgación pueda poner en riesgo la seguridad de la sociedad (vida, seguridad, salud de la población) o del Estado (soberanía nacional, relaciones internacionales, actividades de inteligencia). Por eso, a pesar de ser públicas, el acceso a ellas debe ser restringido por medio de la clasificación de la autoridad competente. Conforme al riesgo que su divulgación puede proporcionar a la sociedad o al Estado, la información pública puede ser clasificada como ultra secreta (plazo de secreto de 25 años, renovable una vez), secreta (con plazo de secreto de 15 años) y reservada (con plazo de secreto de 5 años). La información sigilosa es aquella que otra Leyes protegen como sigilos fiscales, bancarios $\mathrm{o}$ industriales.

El Decreto 7752/2012 ha ampliado la lista de excepciones a la concesión de la información declarando que no serán atendidos los pedidos genéricos; desproporcionados o desrazonados o que exijan trabajos adicionales de análisis, interpretación o consolidación de datos e informaciones, o servicio de producción o tratamiento de datos que no sea de competencia del órgano o entidad. El concepto legal 
de pedido genérico es del pedido que no no describe de forma delimitada (cantidad, período temporal, ubicación, sujeto, recorte temático, formato, etc.) el objeto del pedido, lo que imposibilita la identificación y la comprensión del objeto de la solicitud. Faltan datos importantes para su delimitación y atención.

Los pedidos son considerados desproporcionales y no adecuados, de modo que su atención no comprometa significativamente la realización de las actividades rutinarias de la institución, acarreando perjuicio injustificado a los derechos de otros solicitantes. El órgano debe indicar las razones de hecho o de derecho de la negativa total o parcial de la demanda, presentando el nexo entre la solicitud y los impactos negativos al órgano.

El concepto de pedido desrazonado es aquel que no encuentra amparo para la concesión de acceso solicitado en los objetivos de la LAI y tampoco en sus dispositivos legales, ni en las garantías fundamentales previstas en la Constitución. Es una petición que se caracteriza por la desconformidad con los intereses públicos del Estado en favor de la sociedad, como la seguridad pública, la celeridad y la economía de la administración pública.

Son considerados trabajos adicionales de análisis, interpretación o consolidación de datos e informaciones cuando no hay la información en el formato especificado por el ciudadano, la producción de la información es posible y existe la necesidad de tratamiento de la información. El órgano debe indicar las razones de hecho o de derecho de la negativa total o parcial de la demanda, presentando el nexo entre la solicitud y los impactos negativos al órgano. Son considerados pedidos que necesitan de servicio de producción o tratamiento de datos que no sean de competencia del órgano o entidad cuando el órgano no tiene la información o no es de su competencia.

La LAI también prevé que "el derecho de acceso a los documentos o la información contenida en ellos utilizados como fundamento de la toma de decisión y del acto administrativo se garantizará con la edición del acto de decisión respectivo", es decir, cuando hay un proceso decisorio en curso. En estos casos, el órgano puede negar el acceso a la información, explicando al ciudadano que la información podrá estar disponible después de la conclusión del acto decisorio. Si es posible, el órgano debe indicar una previsión de cuándo se tomará la decisión. Los pedidos de acceso a la información pueden ser hechos por el sistema de acceso a la información (e-sic) o presencialmente, sin 
embargo, todos los pedidos deben ser incluidos en el e-sic, que, por determinación de la “Portaria Interministerial CGU/MPOG 1.254/2015" (BRASIL,CGU,MPOG, 2015), es la única porta de entrada de los pedidos de información del Poder Ejecutivo Federal. Cada órgano debe tener un sector responsable que reciba el pedido de información, ese sector puede funcionar dentro de otro sector pero debe ser llamado de Servicio de Información al Ciudadano - SIC.

Después del pedido, la Ley ha determinado que debe ser autorizado o concedido el acceso inmediato a la información disponible. Caso no disponible, hay un plazo de 20 días para que sean concedidas las informaciones. Ese plazo puede ser prorrogado por más 10 días con justificación. Caso ocurra la recusa a la concesión de la información, la persona que ha hecho el pedido puede recurrir en hasta 10 días de la ciencia de la respuesta. El primer recurso es dirigido al superior jerárquico de la autoridad que ha decidido. El órgano tiene 5 días para decidir el recurso. Caso haga nueva recusa al acceso, el requirente tiene 10 días para recurrir a segunda instancia. El recurso de segunda instancia es decidido por la autoridad máxima del órgano y debe ser decidido también en 5 días. Caso se mantenga la recusa, es posible al demandante impetrar un recurso de tercera instancia. En ese recurso, la autoridad decisora es exterior al órgano, la Contraloría General de la Unión (CGU) debe decidir en 5 días y su decisión vincula el órgano. Si existir otra recusa, el ciudadano puede recurrir a la Comisión Mixta de Revaluación de Informaciones (CMRI) en 10 días que debe contéstalo hasta la $3^{a}$ reunión después del recibimiento del recurso.

Para garantizar el cumplimento de la Ley, dentro del órgano, está prevista la indicación de una autoridad de monitoreo. También corresponde a la autoridad de monitoreo recomendar medidas para perfeccionar las normas y procedimientos necesarios para la efectividad del acceso a la información en la institución. La otra autoridad que garantiza el cumplimento es la CGU, que es responsable por monitorear la Ley en todo Poder Ejecutivo Federal. Es de competencia de la CGU el fomento a la cultura de la transparencia y la concientización sobre el derecho de acceso a la información. Es de responsabilidad también de la CGU publicar informaciones estadísticas sobre la implementación de la Ley de Acceso y preparar informe anual a ser encaminado al Congreso Nacional.

Caso el órgano no conteste el pedido inicial en 30 días, se puede, hasta 10 
días después de ese plazo, impetrar una reclamación que se dirige a la autoridad de monitoreo de LAI . Si la reclamación realizada es infructuosa, el solicitante podrá, además, presentar recurso a la Contraloría General de la Unión, en el plazo de 10 (diez) días contados desde el término del plazo para manifestación por parte de la autoridad de monitoreo. La CGU deberá manifestarse en el plazo de 5 (cinco) días, contado de la recepción del recurso.

Ese conocimiento previo de la LAI será útil para el entendimiento del análisis a ser hecho en el próximo apartado.

\section{ANÁLISIS DEL FUNCIONAMIENTO DE LOS SERVICIOS DE INFORMACIÓN AL CIUDADANO DE ANATEL, ANEEL Y ANP}

En ese apartado será hecho un análisis de la descripción del Sistema de Información al Ciudadano y exámenes cuantitativos y cualitativos de los pedidos de acceso a la información de cada una de las tres agencias: ANATEL, ANEEL y ANP. Será analizada la relación entre los temas demandados por el ciudadano en el e-sic (BRASIL,CGU, 2018) y el estatus del pedido: (i) concedido, (ii) negado (iii) parcialmente negado, (iv) inexistente, (v) órgano sin competencia para responder o (vi) pregunta repetida. Para eso, serán utilizados los datos del e- sic de mayo de 2012 a abril de 2017, con un análisis por año. Así, serán conocidos cuál es el porcentaje de los pedidos de acceso y los temas que no fueron atendidos. Los temas son mapeados y divididos por el Poder Ejecutivo Federal en 17 categorías discriminadas según la tabla siguiente:

Tabla 01: Categorías

\begin{tabular}{c|c}
\hline C1- Agricultura, Extracción y Pesca & Categorías \\
\hline Ciencia, Información & 10. Industria \\
\hline Documentación & 11. Justicia y Legislación \\
\hline 3 Comercio, servicios y Turismo & 12. Medio-Ambiente \\
\hline 4Cultura, Lazer y Deporte & 13. Personas, Familia y Sociedad \\
\hline 5 Defensa y Seguridad & 14. Relaciones Internacionales \\
\hline 6 Economía y Finanzas & 15. Salud \\
\hline 7Educación & 16. Trabajo \\
\hline 8Gobierno y Política & 17. Transporte y Transito \\
\hline Urbanismo Habitación, Saneamiento y & \\
\hline
\end{tabular}

Fuente: BRASIL, (2018) Elaboración propia 
Para caracterización de cada servicio de información al ciudadano, serán utilizados datos referentes al cuestionario de la CGU contestado por los sujetos al decreto 7.724/2012 que ha reglamentado la Ley de acceso a información en el Poder Ejecutivo federal. (BRASIL,CGU, 2017)

\subsection{Agencia Nacional de Telecomunicaciones (ANATEL)}

El Servicio de Información al Ciudadano (SIC) no está previsto en la estructura del órgano lo que es visto como un peligro al acceso a la información porque no hay, en el Regimiento Interno, sector responsable por gestionar los pedidos. Cuanto a la persona que gestiona los pedidos, es un servidor público, lo que, debido a la estabilidad del servidor público brasileño, confiere al SIC una mayor independencia en relación con la administración de la agencia. El SIC no funciona físicamente, en un local de fácil acceso a la población lo que puede inhibir la población a presentar pedidos presenciales y va contra lo que prevé el artículo 9 de la Ley de Acceso a la Información. Otro punto de preocupación es que, mismo después de 6 años de su publicación, la LAI aún no ha sido reglamentada internamente, lo que puede representar una falta de seguridad jurídica. La falta de presupuesto único para el sector y la cultura organizacional que todavía no está alineada con la concesión de la información como regla son apuntados como principales desafíos del SIC. Es destacada también el grande número de solicitaciones que no son de competencia del SIC y si son reclamaciones ordinarias contra las empresas reguladas.

Pasemos ahora a los datos cuantitativos. En cuanto a los números de pedidos, hubo una caída considerable en los últimos 2 años. El tiempo medio de respuesta ha crecido en los periodos de mayo de 2013 a abril de 2014 y de mayo de 2014 a abril de 2015 (también fueron periodos con un número grande de pedidos) pero en el último periodo medido (mayo de 2016 a abril de 2017), ha alcanzado 8,20 días lo que es razonable si considerarnos que, a pesar de la concesión ser inmediata en el caso de disponibilidad de la información, el plazo legal máximo es de 20 días. 
Tabla 02: ANATEL número y tiempo medio

\begin{tabular}{|c|c|c|}
\hline periodo & número de pedidos & tiempo medio de la respuesta (dias) \\
\hline $05 / 2012$ a 04/2013 & 1528 & 9,35 \\
\hline $05 / 2013$ a 04/2014 & 2336 & 12,50 \\
\hline $05 / 2014$ a 04/2015 & 2002 & 13,20 \\
\hline $05 / 2015$ a 04/2016 & 1248 & 7,91 \\
\hline $05 / 2016$ a 04/2017 & 968 & 8,20 \\
\hline
\end{tabular}

Fuente:BRASIL(2018) Elaboración propia

Cuanto a la negación o concesión del número de pedidos de acceso se puede verificar que el porcentaje de pedidos concedidos ha disminuido desde la creación del SIC. Sin embargo, en los periodos con menor concesión de pedidos, hay un gran porcentaje de pedidos que no son de acceso a información, lo que corresponde con lo que está destacado en la descripción del SIC. A pesar del número relativamente bajo de concesión pedidos, son relativamente pocos los pedidos negados.

Tabla 03: ANATEL situación de pedidos

\begin{tabular}{|c|c|c|c|c|c|c|c|}
\hline periodo & $\begin{array}{r}\text { pedidos } \\
\text { concedidos }\end{array}$ & $\begin{array}{l}\text { pedidos } \\
\text { negados }\end{array}$ & $\begin{array}{r}\text { pedidos } \\
\text { parcialmente } \\
\text { concedidos }\end{array}$ & $\begin{array}{l}\text { información } \\
\text { inexistente }\end{array}$ & \begin{tabular}{|r|} 
no es pedido \\
de acceso a ia \\
información \\
\end{tabular} & $\begin{array}{l}\text { órgano } \sin \\
\text { competencia } \\
\text { para responder }\end{array}$ & $\begin{array}{l}\text { pregunta } \\
\text { repetida }\end{array}$ \\
\hline $\begin{array}{l}05 / 2012 a \\
04 / 2013\end{array}$ & $87,238 \%$ & $3,665 \%$ & $1,047 \%$ & 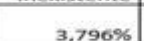 & $0.458 \%$ & $3.534 \%$ & $52 \%$ \\
\hline $05 / 2013$ a & & & & & & & \\
\hline $05 / 2014 a$ & 45,93396 & $0,599 \%$ & 1,58490 & $0,985 \%$ & $46,019 \%$ & $1,292 \%$ & $4 \%$ \\
\hline$\frac{04 / 2015}{05 / 2015 \text { a }}$ & $46,204 \%$ & $0,699 \%$ & $1,898 \%$ & $1,249 \%$ & $47,902 \%$ & $1,299 \%$ & 0,7499 \\
\hline $04 / 2016$ & $50,641 \%$ & $0,401 \%$ & $0,962 \%$ & $2,564 \%$ & $43,590 \%$ & $0,801 \%$ & $1,042 \%$ \\
\hline $\begin{array}{l}05 / 2016 a \\
04 / 2017\end{array}$ & $47,314 \%$ & $1.240 \%$ & $1,343 \%$ & $2,583 \%$ & $44,215 \%$ & $1,446 \%$ & \\
\hline
\end{tabular}

Fuente: BRASIL (2018) Elaboración propia

El porcentaje de pedidos genéricos es la justificativa más declarada para no conceder el acceso. La información sigilosa clasificada conforme la Ley 12.527/2011 es la segunda razón declarada para no conceder acceso a la información.

Tabla 04 ANATEL motivo de negativa

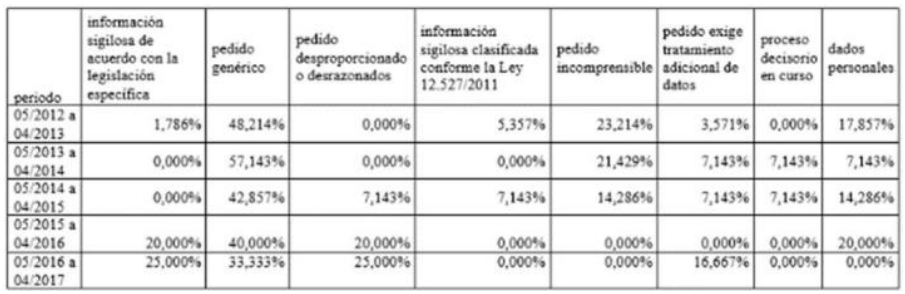

Fuente: BRASIL (2018) Elaboración propia 
En todos los periodos analizados, el tema más demandado fue el de ciencia, información y documentación que, en los dos últimos periodos examinados tuvo más de 90\% de las solicitudes. La tabla abajo contiene solo los temas más demandados, la suma del porcentaje no corresponde a $100 \%$ porque la CGU publica solamente los temas con mayor número de pedidos.

Tabla 05: ANATEL tema de pedido

\begin{tabular}{|l|r|r|r|r|r|r|r|}
\hline $\begin{array}{l}\text { Periodo } \\
\text { Perencia, }\end{array}$ & $\begin{array}{l}\text { comercio, } \\
\text { información y } \\
\text { documentación } \\
\text { información } \\
\text { y turismo }\end{array}$ & $\begin{array}{l}\text { gobierno } \\
\text { y politica }\end{array}$ & $\begin{array}{l}\text { justicia y } \\
\text { legislación }\end{array}$ & $\begin{array}{l}\text { persona, } \\
\text { familia, } \\
\text { sociedad }\end{array}$ & $\begin{array}{l}\text { economía } \\
\text { y finanzas }\end{array}$ & Trabajo \\
\hline $\begin{array}{l}04 / 2012 \text { a } \\
04 / 2013\end{array}$ & $54,91 \%$ & $30,69 \%$ & $6,48 \%$ & $4,19 \%$ & $1,05 \%$ & $0,33 \%$ & $0,00 \%$ \\
\hline $\begin{array}{l}05 / 2013 \\
04 / 2014\end{array}$ & $74,96 \%$ & $8,26 \%$ & $1,07 \%$ & $1,50 \%$ & $11,22 \%$ & $1,76 \%$ & $0,00 \%$ \\
\hline $\begin{array}{l}05 / 2014 \text { a } \\
04 / 2015\end{array}$ & $89,51 \%$ & $0,50 \%$ & $0,90 \%$ & $1,10 \%$ & $5,69 \%$ & $0,60 \%$ & $0,65 \%$ \\
\hline $\begin{array}{l}05 / 2015 \\
04 / 2016\end{array}$ & $92,94 \%$ & $0,00 \%$ & $3,04 \%$ & $0,32 \%$ & $0,80 \%$ & $1,44 \%$ & $0,96 \%$ \\
\hline $\begin{array}{l}05 / 2016 \text { a } \\
04 / 2017\end{array}$ & $94,83 \%$ & $0,00 \%$ & $1,76 \%$ & $0,31 \%$ & $0,72 \%$ & $1,52 \%$ & $0,81 \%$ \\
\hline
\end{tabular}

Fuente: BRASIL (2018) Elaboración propia

Siendo el tema más solicitado, ciencia, información y documentación también fue el tema más negado. A pesar de que en los años estudiados no está entre los temas más solicitados, el tema de persona, familia y sociedad fue uno de los temas que más resultaron en negativas de acceso. Este es resultado de pedidos sobre datos personales que son excepción a la regla de concesión de la información.

Tabla 06: ANATEL tema negación

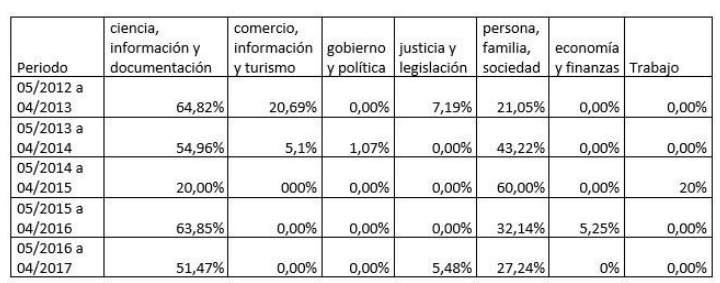

Fuente: BRASIL (2018) Elaboración propia

El hecho que hagan pocos pedidos negados ha resultado en un bajo porcentaje de recursos. De los recursos, pocos (menos de $1 \%$ en todos los periodos) llegan a la cuarta instancia (CMRI). 
Tabla 07: ANATEL Recursos

\begin{tabular}{|l|r|r|r|r|}
\hline periodo & $\begin{array}{r}\text { recurso al jefe } \\
\text { jerárquico }\end{array}$ & $\begin{array}{r}\text { recurso a la autoridad } \\
\text { máxima }\end{array}$ & $\begin{array}{r}\text { recurso a la } \\
\text { CGU }\end{array}$ & $\begin{array}{r}\text { recurso a la } \\
\text { CMRI }\end{array}$ \\
\hline $\begin{array}{l}\text { 05/2012 a } \\
04 / 2013\end{array}$ & $7,330 \%$ & $2,160 \%$ & $1,571 \%$ & $0,000 \%$ \\
\hline $\begin{array}{l}05 / 2013 \\
04 / 2014\end{array}$ & $4,752 \%$ & $1,156 \%$ & $0,557 \%$ & $0,043 \%$ \\
\hline $\begin{array}{l}05 / 2014 \text { a } \\
04 / 2015\end{array}$ & $5,794 \%$ & $1,199 \%$ & $0,350 \%$ & $0,150 \%$ \\
\hline $\begin{array}{l}05 / 2015 \text { a } \\
\text { 04/2016 }\end{array}$ & $8,173 \%$ & $2,404 \%$ & $1,282 \%$ & $0,641 \%$ \\
\hline $\begin{array}{l}05 / 2016 \text { a } \\
04 / 2017\end{array}$ & $6,508 \%$ & $1,033 \%$ & $0,103 \%$ & $0,000 \%$ \\
\hline
\end{tabular}

Fuente: BRASIL (2018) Elaboración propia

\subsection{Agencia Nacional de Energía Eléctrica ( ANEEL)}

Con relación a la previsión del SIC en el organigrama funcional de ANEEL ésta es inexistente, lo que puede ser apuntado como una debilidad de la transparencia activa de la Agencia. Hay cierto grado de seguridad jurídica en la concesión de la información porque la Ley de acceso a la información es reglamentada internamente por la orden de regulación 3836/2016 (BRASIL,ANEEL, 2016). Pasemos ahora a el análisis cuantitativo.

El número de pedidos recibidos por ANEEL ha crecido (más de nueve veces) del periodo de mayo de 2013 a abril de 2014 para el periodo de mayo de 2014 a abril de 2015. Ese aumento no ha generado un tiempo más grande de respuesta, por el contrario, el SIC, a pesar de tener más trabajo, ha disminuido el tiempo de respuesta.

Tabla 08: ANEEL número de pedidos y tiempo medio

\begin{tabular}{|c|c|c|}
\hline periodo & número de pedidos & tiempo medio de la respuesta (dias) \\
\hline $05 / 2012$ a 04/2013 & 716 & 10,13 \\
\hline $05 / 2013$ a 04/2014 & 1081 & 7,16 \\
\hline $05 / 2014$ a 04/2015 & 9959 & 6,51 \\
\hline $05 / 2015$ a 04/2016 & 5034 & 6,16 \\
\hline $05 / 2016$ a 04/2017 & 3634 & 7,04 \\
\hline
\end{tabular}

Fuente: BRASIL (2018) Elaboración propia

Durante todo el periodo examinado, el porcentaje de pedido ha sido mayor que $80 \%$, lo que es razonable, ya que la concesión del acceso a la información es la regla. El porcentaje de pedidos negados no es grande, pero está aumentando desde el periodo de mayo de 2015 a abril de 2015. 
Tabla 09: ANEEL situación de pedidos



Fuente: BRASIL (2018) Elaboración propia

En cuatro de los cinco periodos estudiados el motivo más declarado para la negativa de acceso a la información fue "información sigilosa clasificada conforme la Ley $12.527 / 2011 ”$.

Tabla 10: ANEEL Motivo de negative

\begin{tabular}{|c|c|c|c|c|c|c|c|c|}
\hline & \begin{tabular}{c|} 
información \\
sigilosa de \\
acuerdo con \\
la legislación \\
especifica
\end{tabular} & \begin{tabular}{c|} 
pedido \\
genérico
\end{tabular} & \begin{tabular}{c|} 
pedido \\
desproporcionado \\
dearazonados
\end{tabular} & $\begin{array}{c}\text { información } \\
\text { sizilosa } \\
\text { clasificada } \\
\text { conforme 1a } \\
\text { Ley } \\
12.527 / 2011 \\
\end{array}$ & \begin{tabular}{c|} 
pedido \\
incomprensible
\end{tabular} &  & $\begin{array}{l}\text { proceso } \\
\text { decisorio } \\
\text { en curso }\end{array}$ & $\begin{array}{c}\text { dados } \\
\text { personales }\end{array}$ \\
\hline & 8,0009 & $24,000 \%$ & $0,000 \%$ & 20,00096 & $24,000 \%$ & $24,000 \%$ & $0,000 \%$ & $0,000 \%$ \\
\hline & 5,40596 & 16,21696 & $2,103 \%$ & $13,514 \%$ & $21,622 \%$ & $10,811 \%$ & $29,730 \%$ & $0,000 \%$ \\
\hline & $19,417 \%$ & $7,767 \%$ & $8,738 \%$ & $27,670 \%$ & $2,427 \%$ & $9.223 \%$ & $15.534 \%$ & $9.923 \%$ \\
\hline $\begin{array}{l}05 / 2015 \mathrm{a} \\
042016 \\
052016\end{array}$ & $24,138 \% 6$ & $\begin{array}{r}5,60306 \\
0.9906\end{array}$ & $\frac{6,466 \% 6}{7,4260 \%}$ & $\begin{aligned} 28,44896 \\
32.670^{\circ}\end{aligned}$ & $\frac{6,466^{\circ} 6}{1980^{\circ}}$ & $\begin{array}{l}7,759 \% \\
6.931 \%\end{array}$ & $\begin{array}{l}18,103 \% \\
20,297 \%\end{array}$ & $\begin{array}{l}3,017 \% \\
9406 \%\end{array}$ \\
\hline & & & & & & & & \\
\hline
\end{tabular}

Fuente: BRASIL (2018) Elaboración propia

En todos los periodos analizados, el tema más demandado fue el de gobierno y política, la suma del porcentaje no corresponde a $100 \%$ porque la CGU publica solamente los temas con mayor número de pedidos.

Tabla 11: ANEEL Tema de pedidos

\begin{tabular}{|c|c|c|c|c|c|c|c|c|}
\hline periodo & \begin{tabular}{|l|} 
ciencia \\
información \\
y \\
communicación
\end{tabular} & $\begin{array}{l}\text { comercio, } \\
\text { información y } \\
\text { turismac }\end{array}$ & $\begin{array}{l}\text { gobierno } \\
\text { y politica }\end{array}$ & $\begin{array}{l}\text { justicia } \\
\text { legislación }\end{array}$ & $\begin{array}{l}\text { persona, } \\
\text { familiai, } \\
\text { sociedad }\end{array}$ & $\begin{array}{l}\text { economia } \\
\text { y finanzas }\end{array}$ & Trabajo & Industria \\
\hline $\begin{array}{l}05 / 2012 \text { a } \\
04 / 2013\end{array}$ & $11,87 \%$ & $8,80 \%$ & $49,86 \%$ & $3,77 \%$ & $0,00 \%$ & $3,50 \%$ & $0,00 \%$ & $12,01 \%$ \\
\hline $\begin{array}{l}05 / 2013 \text { a } \\
04 / 2014\end{array}$ & $8,97 \%$ & $8,70 \%$ & $54,21 \%$ & $4,53 \%$ & $0,00 \%$ & $5,28 \%$ & $2,22 \%$ & $9,62 \%$ \\
\hline $\begin{array}{l}05 / 2014 \text { a } \\
04 / 2015\end{array}$ & $16,40 \%$ & $5,02 \%$ & $51,75 \%$ & $0,00 \%$ & $3,63 \%$ & $8,78 \%$ & $0,00 \%$ & $9.26 \%$ \\
\hline${ }_{04 / 2016}^{05}$ & $12,89 \%$ & $0,00 \%$ & $51,91 \%$ & $2,86 \%$ & $4,69 \%$ & $12,12 \%$ & $0,00 \%$ & $12,89 \%$ \\
\hline $\begin{array}{l}05 / 2016 \text { a } \\
04 / 2017 \\
0\end{array}$ & $15,86 \%$ & $0,00 \%$ & $53,05 \%$ & $2,56 \%$ & $2,75 \%$ & $9,50 \%$ & $0,00 \%$ & $13,46 \%$ \\
\hline
\end{tabular}

Fuente: BRASIL (2018) Elaboración propia

De los pedidos negados, el tema con mayor proporción de negativas fue el de ciencia, información y comunicación a pesar de no ser el tema con el mayor número de pedidos. 
Tabla 12: ENEEL Motivo de la negación



Fuente: BRASIL (2018) Elaboración propia

El Servicio de Información al ciudadano tuvo índices muy bajos de recursos desde la implantación del sistema. Eso se debe al bajo porcentaje de negativas de acceso a la información. Merece destaque el hecho de que pocos recursos llegan a la cuarta instancia (CMRI) lo que es un indicio de que la negativa de acceso fue bien basada.

Tabla 13: ANEEL Recursos

\begin{tabular}{|c|c|c|c|c|}
\hline periodo & $\begin{array}{r}\text { recurso al jefe } \\
\text { jerárquico }\end{array}$ & $\begin{array}{r}\text { recurso a la } \\
\text { autoridad máxima } \\
\end{array}$ & $\begin{array}{r}\text { recurso a la } \\
\text { CGU }\end{array}$ & recurso a la CMRI \\
\hline $05 / 2012$ a $04 / 2013$ & $6,285 \%$ & $2,235 \%$ & $0,419 \%$ & $0,000 \%$ \\
\hline $05 / 2013$ a $04 / 2014$ & $6,383 \%$ & $2,960 \%$ & $1,943 \%$ & $0,000 \%$ \\
\hline 05/2014 a 04/2015 & $2,390 \%$ & $0,592 \%$ & $0,171 \%$ & $0,010 \%$ \\
\hline $05 / 2015$ a $04 / 2016$ & $5,761 \%$ & $0,894 \%$ & $0,298 \%$ & $0,099 \%$ \\
\hline 05/2016 a 04/2017 & $8,200 \%$ & $1,486 \%$ & $0,358 \%$ & $0,083 \%$ \\
\hline
\end{tabular}

Fuente: BRASIL (2018) Elaboración propia

\subsection{Agencia Nacional de Petróleo, Gas Natural y Biocombustibles}

No hay previsión del Servicio de Información al Ciudadano en la estructura organizacional de la ANP, lo que puede ser considerada desfavorable a la transparencia. El gestor del SIC no es un servidor público lo que puede causar preocupaciones porque no teniendo estabilidad, es más fácil de no se tener autonomía con relación a la administración y exigir la concesión de la información como regla. Los procedimientos referentes a la Ley de acceso a la información son reglamentados internamente por la Portaría 106/2013 (BRASIL,ANP, 2013), lo que genera seguridad jurídica.

Veamos ahora los datos cuantitativos. En cuanto al número de pedidos, después de disminuir en el período de mayo de 2015 a abril de 2016, hay un crecimiento de solicitaciones registradas. El tiempo medio de las respuestas ha crecido después de una pequeña disminuida en el período de mayo de 2014 a abril de 2015 y ya está en 14,55 días, cerca del límite legal de 20 días. 
Tabla 14: ANP Número y tiempo medio de pedidos

\begin{tabular}{|c|c|c|}
\hline Periodo & Numero de pedidos & tiempo medio de la respuesta (dias) \\
\hline $05 / 2012$ a $04 / 2013$ & 434 & 8,58 \\
\hline $05 / 2013$ a $04 / 2014$ & 512 & 12,16 \\
\hline $05 / 2014$ a $04 / 2015$ & 508 & 14,80 \\
\hline $05 / 2015$ a $04 / 2016$ & 463 & 14,03 \\
\hline $05 / 2016$ a $04 / 2017$ & 551 & 14,55 \\
\hline
\end{tabular}

Fuente: BRASIL (2018) Elaboración propia

Como se puede observar en la tabla 15, la proporción de pedidos concedidos por la ANP ha decrecido entre 05/2012 a 04/2016. El porcentaje del período de 05/2016 a 04/2014 ha aumentado un poco en comparación con el período de 05/2015 a 04/2016 pero continua debajo de los $70 \%$. La proporción de pedidos negados es alta si considerarnos que la concesión de la información es la regla.

Tabla 15: ANP Situación de pedidos

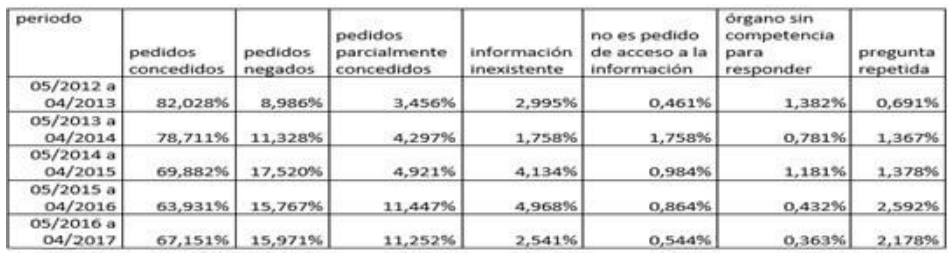

Fuente: BRASIL (2018) Elaboración propia

La información sigilosa (de acuerdo con la legislación específica o con la Ley 12.527/2011) es la principal motivación para la negativa de acceso en ANP desde la implantación del Servicio de Información al Ciudadano. Eso muestra que la concesión de la información es importante pero también hay la necesidad de resguardarse el sigilo.

Tabla 16: ANP motivo de negación

\begin{tabular}{|c|c|c|c|c|c|c|c|c|}
\hline & $\begin{array}{l}\text { Información } \\
\text { sigilosa de } \\
\text { acuerdo con } \\
\text { ia Iegisiación } \\
\text { espocifica }\end{array}$ & $\begin{array}{l}\text { Pedido } \\
\text { generico }\end{array}$ & $\begin{array}{l}\text { Pedido } \\
\text { despropordonado } \\
\text { o desmazonado }\end{array}$ & $\begin{array}{l}\text { Informacion } \\
\text { sigliosa } \\
\text { cias ificada } \\
\text { conforme ia } \\
\text { Ley } \\
12.527 / 2011\end{array}$ & $\begin{array}{l}\text { Pedido } \\
\text { Incomprensibie }\end{array}$ & $\begin{array}{l}\text { Pedido exige } \\
\text { tratamiento } \\
\text { adiclonal de } \\
\text { datos }\end{array}$ & $\begin{array}{l}\text { Proceso } \\
\text { dedsorio } \\
\text { en curso }\end{array}$ & $\begin{array}{l}\text { Dados } \\
\text { personales }\end{array}$ \\
\hline $05 / 2012+04 / 2013$ & $25,641 \%$ & $25,641 \%$ & $0,000 \%$ & $20,513 \%$ & $15,385 \%$ & 7,69276 & $2,564 \%$ & $2,564 \%$ \\
\hline $05 / 2013+04 / 2014$ & $34.489 \%$ & 6,89076 & $17,241 \%$ & 24,13896 & $10,345 \%$ & Q,00066 & $3,448 \% 6$ & $3,448 \%$ \\
\hline $05 / 2014 a 04 / 2015$ & $42,6007 \%$ & 0,00006 & $1,124 \%$ & $38,202 \%$ & $3,371 \%$ & 2,8696 & $5,518 \%$ & $1,124 \%$ \\
\hline $05 / 2015 \times 04 / 2016$ & $21,918 \%$ & 0,00006 & $0,0000 \%$ & $63,014 \%$ & $0,000 \%$ & 8.2198 & $6,8.4095$ & 0,00005 \\
\hline $05 / 2016+24 / 2017$ & $63,6359 \%$ & $2,27 \geqslant \mathrm{A6}$ & $0,000 \%$ & $23,064 \%$ & 4,54596 & 1,13006 & $4,545 \% 6$ & $0,0000 \%$ \\
\hline
\end{tabular}

Fuente: BRASIL (2018) Elaboración propia

Un dato importante acerca del SIC de la ANP es que todos los pedidos de acceso fueron clasificados como ciencia, información y documentación, así, lógicamente, 
todos los pedidos negados fueron de ese tema. Un gran porcentaje de pedidos de acceso no concedidos ha llevado a un número considerable de recursos, así, en el primer año se ha llegado a un porcentaje grande de recursos en primera instancia de recurso mismo presentando el muchos pedidos concedidos. En el tercer año del funcionamiento del SIC, hubo el primer recurso a la CMRI (última instancia de recurso).

Tabla 17: ANP Recursos

\begin{tabular}{|l|r|r|r|r|}
\hline Periodo & $\begin{array}{r}\text { recurso al jefe } \\
\text { jerárquico }\end{array}$ & $\begin{array}{r}\text { recurso a la } \\
\text { autoridad máxima }\end{array}$ & $\begin{array}{r}\text { recurso a la } \\
\text { CGU }\end{array}$ & $\begin{array}{r}\text { recurso a la } \\
\text { CMRI }\end{array}$ \\
\hline $05 / 2012$ a 04/2013 & $10,829 \%$ & $2,765 \%$ & $0,922 \%$ & $0,000 \%$ \\
\hline $05 / 2013$ a 04/2014 & $10,742 \%$ & $3,516 \%$ & $1,172 \%$ & $0,000 \%$ \\
\hline $05 / 2014$ a 04/2015 & $10,630 \%$ & $4,134 \%$ & $1,181 \%$ & $0,394 \%$ \\
\hline $05 / 2015$ a 04/2016 & $8,207 \%$ & $0,864 \%$ & $0,648 \%$ & $0,216 \%$ \\
\hline $05 / 2016$ a 04/2017 & $8,167 \%$ & $0,726 \%$ & $0,000 \%$ & $0,000 \%$ \\
\hline
\end{tabular}

Fuente: BRASIL (2018) Elaboración propia

\section{CONCLUSIÓN}

En el primer apartado del presente, fue concluido que la transparencia es necesaria para la justificación de la existencia de las agencias reguladoras. Vimos que, en Brasil, para que haga transparencia pasiva debe se tener en cuenta que la regla es que todo documento o información es pública, siendo el sigilo una excepción. Así, para garantizar ese derecho fundamental (así considerado en Brasil), fue publicada la Ley de acceso a la información (LAI).

Ese instrumento normativo fue publicado en Brasil en 2011, reglamentado en 2012, y ha previsto la creación del Servicio de Información al Ciudadano (SIC) en cada entidad o órgano que sea sometido a la LAI. Los conceptos importantes y las motivaciones aceptadas para la negativa de acceso a la información (que debe ser una excepcionalidad) fueron presentadas. El SIC es un servicio que garantiza la transparencia pasiva prevista en la Ley 12.527/2011 a través del sistema electrónico e-sic. Llevando en cuenta que las agencias reguladoras deben, para justificar su legitimidad, primar por la transparencia pasiva, fue examinado el Servicio de Información al Ciudadano de las tres primeras agencias brasileñas: ANATEL, ANEEL y ANP.

Del análisis de las descripciones de los Servicios de Información a los Ciudadanos fue posible observar que ningún de los SIC's son previstos en la estructura 
organizacional de las agencias lo que puede ser potencialmente dañino a la transparencia una vez que en la norma que ordena el órgano no prevé un sector responsable por eso llevando a posible incerteza. Fue visto que, en el caso de ANATEL, los procedimientos internos del SIC no tienen seguridad jurídica porque, 6 años después de la publicación de la Ley de acceso a la información, no hay reglamentación interna. Esa misma agencia ha reconocido que no cumple lo establecido en el articulo 9 de la LAI que impone a los órganos que tengan un espacio físico de fácil acceso para que el ciudadano pueda ser atendido personalmente.

Otro factor negativo a se destacar es que, en la ANP, el responsable por garantizar, internamente, el cumplimento de la Ley de acceso no es un servidor público lo que puede indica que ni siempre la administración será presionada a conceder la información. Eso ocurre porque, por no tener la estabilidad de un servidor público, el servidor depende de los gestores de la agencia para continuar en su cargo, no poseyendo la independencia necesaria.

De las agencias examinadas, la que ha recibido el mayor número de pedidos fue ANEEL que, mismo con grande demanda, tuve el menor tiempo medio de respuesta. ANATEL fue la agencia con el menor porcentaje de pedidos negados lo que coaduna con la Ley de acceso y con la cultura de transparencia necesaria en una agencia reguladora en la cual la concesión de la información debe ser la regla. La agencia ANP presenta una grande proporción de pedidos negados, principalmente en los últimos años, lo que puede servir de alerta para un examen más profundizado en cada caso de negativa para que se cumpla el precepto de que la negativa de acceso es una excepción.

No coincidentemente, la agencia con mejor estructura para el Servicio de Atendimiento al Ciudadano, ANEEL, fue la que tuvo mejores resultados cualitativos. La reglamentación del SIC en norma interna y un servidor público como gestor del SIC contribuyen para mejorar la transparencia pasiva porque garantizan seguridad jurídica y autonomía para el sector.

En general, la justificativa más usada por las agencias reguladoras para negar el acceso a la información es de que la información es sigilosa, lo que demuestra que a pesar del acceso a la información ser la regla, se debe seguir lo necesario para se proteger otros bienes jurídicos. El tema más pedido en dos de las agencias reguladoras (ANATEL y ANP) fue el relativo a ciencia, información y documentación. En las tres agencias 
examinadas, ese fue el tema que más ha generado negativas de acceso. Ese hecho, sumado a la justificativa de información sigilosa para negativa al acceso, puede demonstrar que hay documentos e informaciones clasificadas como sigilosas lo que impide la concesión del acceso a la información.

Después de todo lo que fue demostrado en el presente, se puede concluir que las agencias reguladoras están, de una manera general, actuando de una forma transparente en el campo de la transparencia pasiva. Los puntos negativos como la no inserción del SIC en el ordenamiento interno de las agencias debe ser corregido de pronto para que haga mayor seguridad en las decisiones de los integrantes del servicio de información al ciudadano. La ANATEL debe inmediatamente cumplir lo que está dispuesto en la Ley y establecer un local físico para que el ciudadano sea atendido.

Por tanto, siguiendo lo previsto en la LAI y promoviendo la transparencia, las agencias reguladoras estarán, al mismo tiempo, garantizando un derecho fundamental (el acceso a la información) y justificando su existencia como órgano autónomo.

\section{REFERENCIAS}

ABRUCIO, F. L. Os avanços e os dilemas do modelo pós-burocrático: a reforma da administração tração pública à luz da experiência internacional recente. In: PEREIRA, L. C. B.; SPINK, P. Reforma do Estado e administração pública gerencial. Rio de Janeiro: FGV, 1998. p. p.1733-199.

BEALES, H. et al. Government Regulation: The Good, The Bad, \& The Ugly, 2012. Disponivel em: <https://regproject.org/wp-content/uploads/RTP-Regulatory-ProcessWorking-Group-Paper.pdf>. Acesso em: 09 mar. 2018.

BERNARDES, M. B.; SANTOS, P. M.; ROVER, A. J.. . V. 4. N. 3. 7. 2. Ranking das prefeituras da região Sul do Brasil: Uma avaliação a partir de critérios estabelecidos na Lei de Acesso à Informação. Revista de Administração Pública, Rio de Janeiro, v. 49, n. 3, p. 761-792, maio/junho 2015. Disponivel em: <http://dx.doi.org/10.1590/00347612119279 >. Acesso em: 09 mar. 2018.

BRAGA, R. M. Impacto da Lei de Acesso à Informação: os casos da Agência Nacional do Petróleo, Gás Natural e Biocombustíveis e das Agências Reguladoras Federais , 2014. Disponivel em: 
<http://bibliotecadigital.fgv.br/dspace/bitstream/handle/10438/11781/Rodrigo\%20Mend es- Disserta\%C3\%A7\%C3\%A3o-vers\%C3\%A3o\%20final.pdf?sequence=1>. Acesso em: 09 mar. 2018.

BRASIL. Plano Diretor da Reforma do Aparelho do Estado, 1995. Disponivel em: $<$ http://www.biblioteca.presidencia.gov.br/publicacoes-oficiais/catalogo/fhc/planodiretor-da-reforma-do- aparelho-do-estado-1995.pdf $>$.

BRASIL,ANEEL. Portaria 3836. [S.1.]. 2016.

BRASIL,ANP. Portaria 106. [S.1.]. 2013.

BRASIL,CGU. Mapeamento de la Implementación de la LAI. [S.1.]. 2017. BRASIL,CGU. Relatórios estatísticos - CGU. [S.1.]. 2018.

BRASIL,CGU,MPOG. Portaria Interministerial 1254/2015. [S.l.]. 2015. BRASIL,CONGRESSO NACIONAL. Ley 12.527/2011. [S.1.]. 2011.

BRASIL,CONGRESSO NACIONAL. Decreto 7.724/2012. [S.1.]. 2012.

BRITO, N. D. D. C. et al. Análise Descritiva dos Pedidos de Acesso à Informação ao Portal da Transparencia do Governo Federal Brasileiro. Revista da Controladoria-Geral da União, Brasília, v. 9, n. 14, 2017. Disponivel em: <https://ojs.cgu.gov.br/index.php/Revista_da_CGU/article/view/93>.

DÍAZ, J. L. B. Transparencia Administrativa y Cuentas Públicas. In: MACHO, R. G. Ordenación y Transparencia Económica en el Derecho Público y Privado. Madrid: Marcia Pons, 2014. p. 243-265.

ETZIONI, A. Is Transparency the Best Disinfectant? Journal of Political Philosophy, Volu- me 18, Issue 4: 389-404., Washington, v. 18, n. 4, p. 389-404, 2010.

GRAU, N. C. La responsabilización en la nueva gestión pública latinoamericana. CLAD (Centro Latino Americano de Administración para el Desarrollo). Buenos Aires: [s.n.]. 2000. p. 269-327.

GUIMARÃES, J. A. C.; MOLINA, J. C. F.; SANTOS, J. C. G. Direito de Acesso à Informação: uma análise a partir das realidades espanhola e brasileira. Informação \& Sociedade: Estudos, João Pessoa, v. 27, n. 2, p. 49- 62, maio/agosto 2017.

MALARET, E. Regulación Economica, Autoridades Independientes y Transparencia. In: MACHO, R. G. Ordenación y Transparencia Económica en el Derecho Público y Privado. Madrid: Marcia Pons, 2014. p. 126- 178.

MAÑAS, J. L. P. Transparencia y Derecho de Acceso a la Información Pública. Algunas 
Reflexiones en Torno al Derecho de Acceso en la Ley 19/2013, de Transparencia, Acceso a la Información y Buen Gobierno. Revista Catalana de Dret Públic, Barcelona, n. 49, p. 1-19, 2014. Disponivel em:

$<$ http://revistes.eapc.gencat.cat/index.php/rcdp/article/viewFile/10.2436-

20.8030.01.29/n49-pinar-es.pdf>. Acesso em: 09 mar. 2018.

MICHENER, G. FOI laws around the world. Journal of Democracy , v. 22, n. 2, p. 145159, april 2011. NOVELINO, M. Direito Constitucional. 5a. ed. ed. São Paulo: Método, 2011.

ONU. Declaração Universal dos Direitos Humanos, adotada e proclamada pela resolução 217 A (III) da Assembleia Geral das Nações Unidas em 10 de dezembro de 1948, 1948. Disponivel em:

<http://unesdoc.unesco.org/images/0013/001394/139423por.pdf>. Acesso em: 09 mar. 2018.

PEREIRA, L. C. B. Da Administração Pública Burocrática à Gerencial. Revista do Serviço Público, Brasília, v. 47, n. 1, 1996. Disponivel em: <http://www.bresserpereira.org.br/papers/1996/95.AdmPublicaBurocraticaAGerencial.p df>. Acesso em: 10 mar. 2018.

PRADO, O. Agências reguladoras e transparência: a disponibilização de informações pela Aneel, 2006. Disponivel em: <http://www.scielo.br/pdf/rap/v40n4/31599.pdf>.

RAUPP, F. M.; PINHO, J. A. Review of Passive Transparency in Brazilian City Councils. Revista da Administração, São Paulo, v. 51, p. 288-298, 2016.

REZENDE, F. A. A Reforma do Estado em perspectiva comparada. In: NASSUNO, M.; KAMADA, P. REZENDE, F. A. Reforma do Estado em perspectiva comparada. In: NASSUNO, M.; KAMADA, P. (Orgs.). Balanço da reforma do Estado no Brasil: a nova gestão pública. Brasí- lia: Seges, 2002. Brasília: Seges, 2002. p. 223-233.

SANTANA, A. Agências Ejecutivas y Agências Reguladoras - O processo de Agencificação: pressupostos do modelo brasileiro e balançao da experiência. In: NASSUNO, M.; KAMADA, P. Balanço da Reforma Administrativa no Brasil. Brasília: Seges, 2002. p. $75-84$. 